\title{
Stability Analysis and Hadamard Synergic Control for a Class of Dynamical Networks*
}

\author{
Xinjin Liu, Yun Zou \\ School of Automation, Nanjing University of Science and Technology, Nanjing, China \\ E-mail: liuxinjin2006@163.com,zouyun@vip.163.com \\ Received February 9, 2010; revised March 21, 2010; accepted June 28, 2010
}

\begin{abstract}
Hadamard synergic control is a new kind of control problem which is achieved via a composite strategy of the state feedback control and the direct regulation of the part of connection coefficients of system state variables. Such a control is actually used very often in the practical areas. In this paper, we discuss Hadamard synergic stabilization problem for a class of dynamical networks. We analyze three cases: 1) Synergic stabilization problem for the general two-node-network. 2) Synergic stabilization problem for a special kind of networks. 3) Synergic stabilization problem for special kind of networks with communication time-delays. The mechanism of the synergic action between two control strategies: feedback control and the connection coefficients regulations are presented.
\end{abstract}

Keywords: Hadamard Synergic Control, Algebraically Graph Theory, Decentralized Feedback Control, Connection Coefficient Gain Matrix

\section{Introduction}

Complex networks of dynamic agents have attracted great interesting in recently years. This is partly due to broad applications of multiagent systems in many areas including physicists, biologists, social scientists and control scientists [1-3], distributed sensor networks [4], and congestion control in communication networks [5] and so on. In fact, a complex dynamical network can be viewed as a large-scale system with special interconnections among its dynamical nodes from a system-theoretic point of view and when we solved the control problems of electric power systems, socioeconomic systems, etc., large-scale interconnected systems with many state variables often appear. In order to stabilize large-scale interconnected systems via the local feedback, the traditional methods usually ignore or try to reduce the influence of interconnections under the condition that the subsystems are controllable. The interconnections among subsystems in large-scale systems are thought to be one of the most important roots to produce complexity recently [6]. To enhance the effects of stabilization, the strategy of coupling two decoupled subsystems via designing a suitable combined feedback are considered in $[7,8]$, which is

${ }^{*}$ This work was supported in part by the National Natural Science Foundation of P. R. China under Grant 60874007 and the Research Fund for the Doctoral Program of Higher Education 200802550024. called the harmonic control.

Along the development of society, interconnections play more and more important roles in social systems, economic systems, power systems, etc. The connections of the system states are a type of the most important structures of a system. In fact, in many fields and even in our daily life, besides the usual feedback controls, it is also very useful for us to control our business by regulating the connections among the subsystems directly. For examples, the damages in power and transportation ties is one of the main facts to result in the huge loss in the freeze disaster in several provinces in southern China in 2008, and reflects the effects of the connections of the subsystems for the social large-scale system; the strict and active quarantine and isolation measures among regions in the SARS and H1N1 is also an example. In fact, in our daily life, we always deal with the interpersonal relationship between ourselves and those around us and the inter-relations between ourselves and the collective around us. Therefore, we can say that the human world is a complex network system through these relationships, and the handling of these relationships is actually the regulation of the connection among persons.

The consideration of the connection problem were mainly seen in the power system research early times, including the transient stability analysis [9] and splitting control [10-12]. The interconnection coefficients of the 
state variables of the subsystems are considered as the control variables that are regulated directly in the splitting control for power system. Furthermore, the isolation treatment strategy is further discussed in the emergency control [13-15]. The studies give a theoretical interpretation for the practical experiences that the early quarantine and isolation strategies are critically important to control the outbreaks of epidemics. Finally, a new kind of concept called Hadamard synergic control is introduced based on the Hadamard matrix product [16]. It is achieved via a composite strategy of the state feedback control and the direct regulation of the part of connection coefficients of system state variables. Such a control improves the limitations of the traditional feedback control [17-19] and may be of some potential applications in the emergency treatment such as isolation and obstruction control. For clear, we give this model again here.

Consider the following linear time-invariant system:

$$
\dot{x}(t)=A x(t)+B u(t)
$$

Here, $A=\left[a_{i j}\right]_{n \times n}, B=\left[b_{i}\right]^{T}, b_{i} \in R^{m}, i=1,2, \cdots, n$

Obviously, the element $a_{i j}$ is the interconnection coefficient between the $i$-th state and the $j$-th state, for convenience, we call the system matrix $A$ as system interconnection matrix. In many practical cases, such as the switches and circuit breakers in the power systems, firewall in the Internet etc., the system interconnection matrix $A$ can be directly regulated, of course, can be pre-designed in some extent. Thus, the system interconnection matrix $A$ can be divided into two parts: $A_{1}+A_{2}$. $A_{1}$ is the fixed part of $A$ which is not able to be regulated directly and $A_{2}$ is the flexible part of $A$ which can be regulated directly in some extent. By using the Hadamard matrix product, this direct regulation of the interconnection matrix $A$ can be written as follows:

$$
A_{K}=A_{1}+A_{2} \circ K
$$

Here, for convenience, we call $K=\left[k_{i j}\right]_{n \times n}$ as the connection coefficient gain matrix. It may be need to satisfy some constraints such as $0 \leq k_{i j} \leq 1$ etc. Of course, the control strategy above is different from the feedback control. $A_{2} \circ K$ is the Hadamard matrix product defined as [20]:

$$
A_{2} \circ K=\left[\tilde{a}_{i j} k_{i j}\right]_{n \times n}, A_{2}=\left[\tilde{a}_{i j}\right]_{n \times n}
$$

Then, the general feedback control problem formulation can be extended as follows: find direct connection coefficient gain matrix $K$ and feedback gain matrix $F$ such that the generalized closed loop system

$$
\dot{x}=\left(A_{1}+A_{2} \circ K+B F\right) x
$$

is stable, robust stable, or some other specific perform- ances. For convenience, we call this kind of control strategy as Hadamard synergic control.

In order to illustrate the idea of isolation and obstruction of the connections among subsystems, we give the following examples [21].

Example 1. Replacing the scalars $a_{i j}, b_{i}, f_{j}, k_{i j}$ by matrices $A_{i j}, B_{i}, F_{j}, k_{i j} E_{i j}$ with appropriate dimensions and the self-loops are not allowed, the general Hadamard synergic control model (3) can be rewritten as:

$$
\begin{aligned}
& \dot{x}_{1}=A_{11} x_{1}+k_{12} A_{12} x_{2}+\cdots+k_{1 n} A_{1 n} x_{n}+B_{11} u_{1} \\
& \dot{x}_{2}=A_{22} x_{2}+k_{21} A_{21} x_{1}+\cdots+k_{2 n} A_{2 n} x_{n}+B_{22} u_{2} \\
& \cdots \cdots \cdots \\
& \dot{x}_{n}=A_{n n} x_{n}+k_{n 1} A_{n 1} x_{1}+\cdots+k_{n n-1} A_{n n-1} x_{n-1}+B_{n n} u_{n}
\end{aligned}
$$

Where $x_{i} \in R^{n_{i}}, i=1,2, \cdots, n$ is the local state of the $i$-th subsystem. $k_{i j}$ are the control variables. System model above can be rewritten as:

$$
\dot{x}=\left(A_{1}+A_{2} \circ K\right) x+B u
$$

Here,

$$
\begin{aligned}
& A_{1}=\left[\begin{array}{lll}
A_{11} & & \\
& \ddots & \\
& & A_{n n}
\end{array}\right], A_{2}=\left[\begin{array}{cccc}
0 & A_{12} & \cdots & A_{1 n} \\
A_{21} & 0 & \cdots & A_{2 n} \\
\vdots & \vdots & \ddots & \vdots \\
A_{n 1} & A_{n 2} & \cdots & 0
\end{array}\right] \\
& B=\left[\begin{array}{cccc}
B_{11} & & \\
& \ddots & \\
0 & k_{12} E_{12} & \cdots & k_{1 n} E_{1 n} \\
k_{21} E_{21} & 0 & \cdots & k_{2 n} E_{2 n} \\
\vdots & \vdots & \ddots & \vdots \\
k_{n 1} E_{n 1} & k_{n 2} E_{n 2} & \cdots & 0
\end{array}\right], K=[1]_{n_{i} \times n_{j}}
\end{aligned}
$$

In power systems, the model (4) can be used to describe the frequency control in multi-areas loads with the balance of active powers among the networks of different areas.

Example 2. Consider the network model researched in $[1,3]$, in fact, the system interconnection matrix $A$ is divided into two parts. By the Kronecker product, this network model can be written as [22]:

$$
\dot{x}=\left(I_{n} \otimes A_{O}+C \otimes A_{C}\right) x+\left(I_{n} \otimes B_{1}\right) u
$$

Where $x=\left(x_{1}^{T}, x_{2}^{T}, \cdots, x_{n}^{T}\right)^{T}, u=\left(u_{1}^{T}, u_{2}^{T}, \cdots, u_{n}^{T}\right)^{T}, C \in R^{n \times n}$ is called as the outer coupling matrix, $A_{C}$ is the inner coupling matrix describing the interconnections.

Obviously, the network model (5) is a special case of the model (4). From the definition of the matrix Kronecker product we know, the connection matrix of the system has very symmetrically consistency structure if we describe the system by using the corresponding Hadamard product, this is: any two subsystems have the same basic 
connection structure except the coupling coefficient, i.e.

$$
\begin{aligned}
& A_{i j}=\left\{\begin{array}{lc}
A_{O} & i=j=1,2, \cdots, n \\
A_{C} & 1 \leq i \neq j \leq n
\end{array}\right. \\
& B_{11}=B_{22}=\cdots=B_{n n}
\end{aligned}
$$

Network model (5) has very specific project background such as in the consensus and formation control problem. This also illustrates the rationality and generality of the abstract model (4).

Example 3. The host population consists of six sub populations: namely susceptible individuals $x_{1}(t)$, asymptomatic individuals $x_{2}(t)$, quarantined individuals $x_{3}(t)$, symptomatic individuals $x_{4}(t)$, isolated individuals $x_{5}(t)$, recovered individuals $x_{6}(t)$. The total population size is $N=\sum_{i=1}^{6} x_{i}(t)$. The detailed descriptions of other parameters see [23]. The SARS transmission model with quarantine and isolation controls $u$ and $v$ is given by the following nonlinear system of differential equations:

$$
\begin{aligned}
& \dot{x}_{1}=\Pi-\frac{\beta x_{4}+\xi_{E} \beta x_{2}+\xi_{Q} \beta x_{3}+\xi_{J} \beta x_{5}}{N}-\mu x_{1} \\
& x_{2}=p+\frac{\beta x_{4}+\xi_{E} \beta x_{2}+\xi_{Q} \beta x_{3}+\xi_{J} \beta x_{5}}{N}-\left(k_{1}+\mu-u\right) x_{2} \\
& \dot{x}_{3}=u x_{2}-\left(k_{2}+\mu\right) x_{3} \\
& \dot{x}_{4}=k_{1} x_{2}-\left(d_{1}+\delta_{1}+\mu\right) x_{4}-v x_{4} \\
& \dot{x}_{5}=v x_{4}-k_{2} x_{3}-\left(d_{2}+\delta_{2}+\mu\right) x_{5} \\
& \dot{x}_{6}=\delta_{1} x_{4}+\delta_{2} x_{5}-\mu x_{6}
\end{aligned}
$$

Obviously, the model above is a typical interconnection-regulation control of a nonlinear system with the control variables $u$ and $v$ (see Figure 1).

Hadamard product is a classical matrix product. It has many applications in some areas especially in mathematics and physics. It also has some applications in signal processing [24]. In the existing literatures, almost all the results about the eigenvalue estimations on Hadamard products were obtained under the presupposition that the involved matrices are special ones such as M-matrices, Hermitian (or the form of $A^{*} A$ ), diagonal matrices, etc. See [25-29] and the other corresponding references. Also, the discussions on the mixture products like $A(B \circ C)$ are scarcely reported. Hence, the basic properties and expressions on Hadamard product still remain to be extensively studied.

Although almost all the existing control theory and applications are implemented by feedback controls, the feedback is, in a general sense, only one of the specific measures to implement the regulations of the connections of system states.

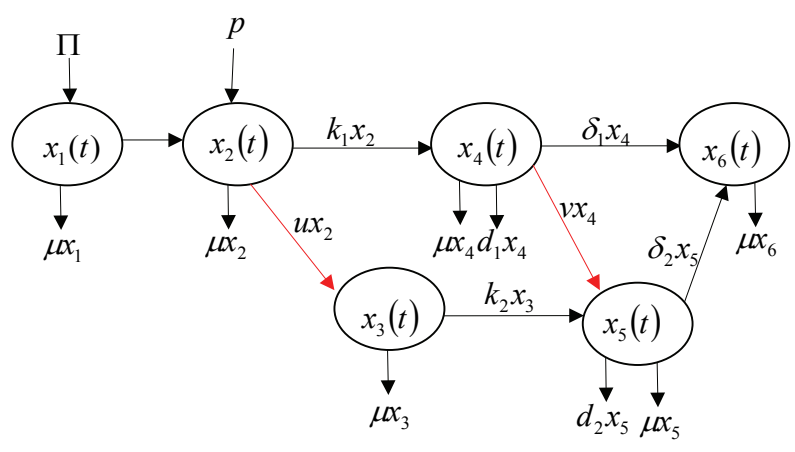

Figure 1. A schematic representation of the populations flow.

Let the feedback law be $u=F x, F=\left[f_{j}\right] \in R^{m \times n}$, $f_{j} \in R^{m}, j=1,2, \cdots, n$. Then the system matrix of the closed loop is of the form: $A=\left[a_{i j}+b_{i}^{T} f_{j}\right]_{n \times n}$. The actual functions of the feedback are the compensations of $a_{i j}$, i.e., regulating the interconnection coefficients from $a_{i j}$ to $a_{i j}+b_{i}^{T} f_{j}$ via the input information channel. Hence, in an open-loop viewpoint, the feedback control is just a special indirect regulation of interconnections of system states.

The observation above show that the feedback control strategy is only one of the specific measures to implement the regulations of the connections of system states via the input information channel, rather than the direct physical regulation of the system interconnection matrix A.

In this paper, we mainly discuss the Hadamard synergic stabilization problem for the general two-node-network (4), and then Hadamard synergic stabilization problem for the special model (5) is studied. Matrix algebra and algebraic graph theory are proved useful tools in modeling the communication network and relating its topology to the discussion of the network stability.

The rest of this paper is organized as follows. System models and problem formulation discussed in this paper are given in Section 2. Hadamard synergic stabilization problem for the general two-node-network (4) is studied in Section 3. In Section 4, Hadamard synergic stabilization problem for the special network model (5) is discussed. Furthermore, networks with communication time-delays are investigated. The last section concludes the paper.

\section{System Models and Problem Formulation}

In this section, we give the system models and problem formulation discussed in this paper.

Because of the existence of the Hadamard product, 
this makes that the stability analysis of the general network model (4) become difficult. Therefore, we mainly consider the Hadamard synergic control problem for the special model (5). Furthermore, Hadamard synergic control for the two-node-network model of the general network system (4) is investigated simply. For convenience, the network model (5) can be rewritten as:

$$
\dot{x}=\left(I_{n} \otimes A_{O}+K \otimes A_{C}\right) x+\left(I_{n} \otimes B_{1}\right) u
$$

$K=\left[k_{i j}\right]_{n \times n}$ is the connection coefficient gain matrix.

In the general case, the control variables $k_{i j}$ often need to satisfy some constraints. There exist the following cases being researched.

Case 1: $k_{i j}$ is discrete. For example $k_{i j}=0,1$. When $k_{i j}=0$, it means that we cut off the connections from the $j$-th subsystem to the $i$-th subsystem; When $k_{i j}=1$, it means that we keep the corresponding connections. In this case, the control is called the isolation treatment strategy. In fact, this kind of control strategy has been researched in many literatures [15] especially in the power electrical engineering [10-12].

Case 2: $k_{i j}$ is continuous. It often needs to meet some constraints. For example, $0 \leq k_{i j} \leq 1$ in the epidemic control $[13,14,25] ; \quad k_{i i}=-\sum_{j \neq i}^{n} k_{i j}$ in the consensus or formation control problem [30,31], etc.

Although the control variables $k_{i j}$ often need to satisfy some constraints, as the stability research in the classical feedback control of the system (1) required to unconstrained control $u(t)$ we also suppose that the connection coefficient $k_{i j} \in R$ in this paper.

We present the formulations of the Hadamard synergic stabilization problems as follows:

Hadamard synergic stabilization problem (HSSP) [21]: Given system (1), and let $A+A_{1}+A_{2}$. Find connection coefficient gain matrix $K \subseteq R^{n \times n}$ and feedback control $u=F x$ such that the corresponding Hadamard synergic closed loop (3) is stable, i.e.

$$
\lambda\left(A_{1}+A_{2} \circ K+B F\right) \subset C^{-}
$$

Where $\lambda($.$) represents the set of eigenvalues of the$ corresponding matrix, $C^{-}$means the left-half complex plane. For convenience, we call the matrix pair $(K, F)$ as the synergic control matrix pair.

Remark 1. Obviously, the HSSP is equivalent to the problem that is to find connection coefficient gain matrix $K$ such that $\left(A_{1}+A_{2} \circ K, B\right)$ is stabilizable. One of the stronger conditions of it is to find a matrix $K$ such that $\left(A_{1}+A_{2} \circ K, B\right)$ is completely controllable. Also, for convenience, we call these two problems as Hadamard synergic stabilization and Hadamard synergic Controllabilization problems respectively.

In this paper, we mainly consider the Hadamard synergic stabilization problem for the two cases:

Case 1: The two-node-network model of the general network system (4).

Case 2: The special network model (6).

\section{Hadamard Synergic Stabilization for THE General Two-Node-Network}

In this section, we consider the Hadamard synergic Stabilization problem for the general dynamical network model (4). We mainly consider the two-node-network described as:

$$
\begin{aligned}
& \dot{x}_{1}=A_{11} x_{1}+\alpha_{12} A_{12} x_{2}+B_{11} u_{1} \\
& \dot{x}_{2}=A_{22} x_{2}+\alpha_{21} A_{21} x_{1}+B_{22} u_{2}
\end{aligned}
$$

Then, the Hadamard synergic stabilization problem for the network model (7) can be presented as: find connection coefficients $\alpha_{12}, \alpha_{21} \in R$ and decentralized feedback control $u_{1}=F_{1} x_{1}, u_{2}=F_{2} x_{2}$ such that the closed loop matrix

$$
A_{\text {loop }}(2)=\left[\begin{array}{cc}
A_{11}+B_{11} F_{1} & \alpha_{12} A_{12} \\
\alpha_{21} A_{21} & A_{22}+B_{22} F_{2}
\end{array}\right]
$$

is stable.

When $\alpha_{12}=0$ or $\alpha_{21}=0$, the stability of $A_{\text {loop }}(2)$ is equal to the stability of the two subsystems, so we do not consider this condition. In the following, we suppose that $\alpha_{12} \neq 0, \alpha_{21} \neq 0$.

\subsection{Case of $\operatorname{rank}\left(A_{12}\right)=\operatorname{rank}\left(A_{21}\right)=1$}

In this section, we discuss the Hadamard synergic stabilization problem of the network model (7) with the special case $\operatorname{rank}\left(A_{12}\right)=\operatorname{rank}\left(A_{21}\right)=1$.

Based on the theorem of the Linear Algebra, let $A_{12}=a_{1} b_{2}^{T}, A_{21}=a_{2} b_{1}^{T}, a_{1}, b_{1}, a_{2}, b_{2} \in R^{n}$. Then, the system (7) without local input can be rewritten as:

$$
\begin{aligned}
& \dot{x}_{1}=A_{11} x_{1}+\alpha_{12} a_{1} b_{2}^{T} x_{2} \\
& \dot{x}_{2}=A_{22} x_{2}+\alpha_{21} a_{2} b_{1}^{T} x_{1}
\end{aligned}
$$

Note that system above is equivalent to the following system:

$$
\begin{array}{ll}
\dot{x}_{1}=A_{11} x_{1}+\alpha_{12} a_{1} y_{2} & y_{1}=b_{1}^{T} x_{1} \\
\dot{x}_{2}=A_{22} x_{2}+\alpha_{21} a_{2} y_{1} & y_{2}=b_{2}^{T} x_{2}
\end{array}
$$

Let $\tilde{u}_{1}=y_{2}, \tilde{u}_{2}=y_{1}$ are the inputs of the intercon- 
nected control; $y_{1}, y_{2}$ are the outputs of the first and second subsystem respectively. Then $\alpha_{12} a_{1}, \alpha_{21} a_{2}$ can be viewed as the matrix of the first and second subsystem accepting the interconnected control respectively. Hence, let

$$
\begin{aligned}
& H_{1}(s)=\alpha_{12} b_{1}^{T}\left(s I-A_{11}\right)^{-1} a_{1} \\
& H_{2}(s)=\alpha_{12} b_{2}^{T}\left(s I-A_{22}\right)^{-1} a_{2}
\end{aligned}
$$

Then, the matrix $A(2)=\left[\begin{array}{cc}A_{11} & \alpha_{12} A_{12} \\ \alpha_{21} A_{21} & A_{22}\end{array}\right]$ can be viewed as the state matrix of the closed-loop feedback system as shown in Figure 2.

Theorem 1. There exist $\alpha_{12}, \alpha_{21} \in R$ such that the matrix $A(2)$ is stable if and only if there exist $\alpha_{12}$, $\alpha_{21} \in R$ such that the polynomial $f(s)=d_{1}(s) d_{2}(s)-$ $\alpha_{12} \alpha_{21} f_{1}(s) f_{2}(s)$ is stable. In this case, the matrices $A_{12}$, $A_{21}$ must satisfy that $\operatorname{tr}\left(A_{12}\right)+\operatorname{tr}\left(A_{21}\right)<0$.

Here,

$$
\begin{aligned}
& d_{1}(s)=\operatorname{det}\left(s I-A_{11}\right), f_{1}(s)=b_{1}^{T}\left(s I-A_{11}\right)^{*} a_{1} \\
& d_{2}(s)=\operatorname{det}\left(s I-A_{22}\right), f_{2}(s)=b_{2}^{T}\left(s I-A_{22}\right)^{*} a_{2}
\end{aligned}
$$

$\operatorname{tr}(),.()^{*}$ denote the trace and adjoins of the corresponding matrix respectively.

Proof. From the analysis above we know that $A(2)$ is stable if and only if the feedback system shown in Figure 2 is stable, where the closed loop transfer function in Figure 2 is

$$
\begin{aligned}
H(s) & =\frac{H_{1}(s)}{1-H_{1}(s) H_{2}(s)} \\
& =\frac{\operatorname{det}\left(s I-A_{11}\right) b_{2}^{T}\left(s I-A_{22}\right)^{*} a_{2}}{d_{1}(s) d_{2}(s)-\alpha_{12} \alpha_{21} f_{1}(s) f_{2}(s)}
\end{aligned}
$$

Therefore, $A(2)$ is stable if and only if the polynomial $d(s)$ is stable. Let $A_{11} \in R^{n_{1} \times n_{1}}, A_{22} \in R^{n_{2} \times n_{2}}$. Then, note that $d(s)$ and $f_{1}(s) f_{2}(s)$ are the polynomials with degree $n_{1}+n_{2}$ and $n_{1}+n_{2}-2$ respectively. Hence, if we let $d(s)=s^{n_{1}+n_{2}}-c s^{n_{1}+n_{2}-1}+d_{0}(s)$, then we have that $c=\operatorname{tr}\left(A_{12}\right)+\operatorname{tr}\left(A_{21}\right)<0$. This completes the proof.

Remark 2. When $\operatorname{rank}(M)=1$, there exist vectors $a, b \in R^{n}$ such that $M=a b^{T}$ and the different decompositions are unique up to a constant, so the result above is independent of the decompositions of the coupled matrices $A_{12}, A_{21}$. The results above can be generalized to cases of multiple subsystems simply.

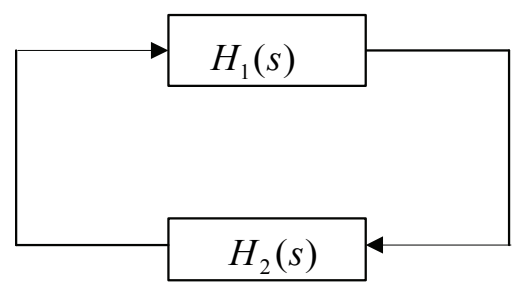

Figure 2. The closed loop of the system (8).

\subsection{Case of $\operatorname{rank}\left(A_{12}\right) \geq 1, \operatorname{rank}\left(A_{21}\right) \geq 1$}

In this section, we discuss the Hadamard synergic stabilization problem of the network model (7) with the general case $\operatorname{rank}\left(A_{12}\right) \geq 1, \operatorname{rank}\left(A_{21}\right) \geq 1$ by using the small gain theorem.

Decompose $A_{12}=B_{1} C_{2}, A_{21}=B_{2} C_{1}$, then $\alpha_{12} A_{12}=$ $\left(\alpha_{12} B_{1}\right) C_{2}, \quad \alpha_{21} A_{21}=\left(\alpha_{21} B_{2}\right) C_{1}$. Let

$$
\begin{aligned}
& H_{1}(s)=C_{1}\left(s I-A_{11}\right)^{-1}\left(\alpha_{12} B_{1}\right) \\
& H_{1}(s)=C_{1}\left(s I-A_{11}\right)^{-1}\left(\alpha_{12} B_{1}\right)
\end{aligned}
$$

Similarly as in the section 3.1 , the matrix $A(2)$ can be viewed as the state matrix of the closed-loop feedback system as shown in Figure 2. In this way, $A_{\text {loop }}(2)$ can be viewed as an interconnected system composing of two subsystems $\left(A_{11}, \alpha_{12} B_{1}, C_{1}\right),\left(A_{22}, \alpha_{21} B_{2}, C_{2}\right)$ under the local feedback.

Using the small gain theorem, we can get the following result.

Proposition 1. If there exist $\alpha_{12}, \alpha_{21}, F_{1}, F_{2}$ such that

$$
\begin{aligned}
& \left\|C_{1}\left(s I-A_{11}-B_{11} F_{1}\right)^{-1}\left(\alpha_{12} B_{1}\right)\right\|_{\infty} \\
& \times\left\|C_{2}\left(s I-A_{22}-B_{22} F_{2}\right)^{-1}\left(\alpha_{21} B_{2}\right)\right\|_{\infty}<1
\end{aligned}
$$

then the system (7) can be stabilized by the synergic control.

In the following, we suppose that

$$
\begin{aligned}
& \alpha_{12} \in\left[\beta_{1}, \gamma_{1}\right], \alpha_{21} \in\left[\beta_{2}, \gamma_{2}\right], \\
& \gamma_{1}>\beta_{1}>0, \gamma_{2}>\beta_{2}>0
\end{aligned}
$$

Remark 3. Based on the Proposition 1, we know that if there exist $\alpha_{12}, \alpha_{21}$ such that

$$
\begin{aligned}
& \left\|C_{1}\left(s I-A_{11}\right)^{-1}\left(\alpha_{12} B_{1}\right)\right\|_{\infty} \\
& \times\left\|C_{2}\left(s I-A_{22}\right)^{-1}\left(\alpha_{21} B_{2}\right)\right\|_{\infty}<1
\end{aligned}
$$

then $A(2)$ is stable. Obviously, there exist $\alpha_{12}, \alpha_{21}$ as in (10) such that (11) holds if and only if 


$$
\begin{aligned}
& \left\|C_{1}\left(s I-A_{11}\right)^{-1}\left(B_{1}\right)\right\|_{\infty} \\
& \times\left\|C_{2}\left(s I-A_{22}\right)^{-1}\left(B_{2}\right)\right\|_{\infty}<\frac{1}{\beta_{1} \beta_{2}}
\end{aligned}
$$

But the decompositions of $A_{12}, A_{21}$ are general not unique.

In the following, we give the suitable decompositions and the explain (12) by LMI method by using the result in [32].

Theorem 2. For any fixed full rank decompositions $A_{12}=B_{1}^{0} C_{2}^{0}, A_{21}=B_{2}^{0} C_{1}^{0}$, there exist connection coefficients $\alpha_{12}, \alpha_{21}$ as in (10) and decompositions $A_{12}=B_{1} C_{2}$, $A_{21}=B_{2} C_{1}$ such that (11) holds if and only if there exist positive matrices $P_{1}, P_{2}, X, Y$ such that

$$
\begin{aligned}
& {\left[\begin{array}{cc}
P_{1} A_{11}+A_{11}^{T} P_{1}+B_{1}^{0} X B_{1}^{0 T} & P_{1} C_{1}^{0 T} \\
C_{1}^{0} P_{1} & -\frac{1}{\beta_{2}^{2}} Y
\end{array}\right]<0} \\
& {\left[\begin{array}{cc}
P_{2} A_{22}+A_{22}^{T} P_{2}+B_{2}^{0} Y B_{2}^{0 T} & P_{2} C_{2}^{0 T} \\
C_{2}^{0} P_{2} & -\frac{1}{\beta_{1}^{2}} X
\end{array}\right]<0}
\end{aligned}
$$

Proof. Based on the result in [32], we know for any fixed full rank decompositions $A_{12}=B_{1}^{0} C_{2}^{0}, A_{21}=B_{2}^{0} C_{1}^{0}$, there exist connection coefficients $\alpha_{12}, \alpha_{21}$ and decompositions $A_{12}=B_{1} C_{2}, A_{21}=B_{2} C_{1}$ such that (11) holds if and only if there exist positive matrices $P_{1}, P_{2}, \tilde{X}, \tilde{Y}$ such that

$$
\begin{aligned}
& {\left[\begin{array}{cc}
P_{1} A_{11}+A_{11}^{T} P_{1}+\alpha_{12}^{2} B_{1}^{0} X B_{1}^{0 T} & P_{1} C_{1}^{0 T} \\
C_{1}^{0} P_{1} & -\tilde{Y}
\end{array}\right]<0} \\
& {\left[\begin{array}{cc}
P_{2} A_{22}+A_{22}^{T} P_{2}+\alpha_{21}^{2} B_{2}^{0} Y B_{2}^{0 T} & P_{2} C_{2}^{0 T} \\
C_{2}^{0} P_{2} & -\tilde{X}
\end{array}\right]<0}
\end{aligned}
$$

Let $X=\alpha_{12}^{2} \tilde{X}, \quad Y=\alpha_{21}^{2} \tilde{Y}$, then the inequalities above can translate into

$$
\begin{aligned}
& {\left[\begin{array}{cc}
P_{1} A_{11}+A_{11}^{T} P_{1}+B_{1}^{0} X B_{1}^{0 T} & P_{1} C_{1}^{0 T} \\
C_{1}^{0} P_{1} & -\frac{1}{\alpha_{21}^{2}} Y
\end{array}\right]<0} \\
& {\left[\begin{array}{cc}
P_{2} A_{22}+A_{22}^{T} P_{2}+B_{2}^{0} Y B_{2}^{0 T} & P_{2} C_{2}^{0 T} \\
C_{2}^{0} P_{2} & -\frac{1}{\alpha_{12}^{2}} X
\end{array}\right]<0}
\end{aligned}
$$

If there exist positive matrices $P_{1}, P_{2}, X, Y$ such that (13) holds, and we can choose connection coefficient $\alpha_{12}=\beta_{1}, \alpha_{21}=\beta_{2}$ such that (11) holds.
Conversely, if there exist connection coefficients $\alpha_{120}, \alpha_{210}$ as in (10) such that (11) holds, then we can get:

$$
\begin{aligned}
& {\left[\begin{array}{cc}
P_{1} A_{11}+A_{11}^{T} P_{1}+B_{1}^{0} X B_{1}^{0 T} & P_{1} C_{1}^{0 T} \\
C_{1}^{0} P_{1} & -\frac{1}{\alpha_{210}^{2}} Y
\end{array}\right]<0} \\
& {\left[\begin{array}{cc}
P_{2} A_{22}+A_{22}^{T} P_{2}+B_{2}^{0} Y B_{2}^{0 T} & P_{2} C_{2}^{0 T} \\
C_{2}^{0} P_{2} & -\frac{1}{\alpha_{120}^{2}} X
\end{array}\right]<0}
\end{aligned}
$$

By using Schur complement, we know that the inequalities above are equal to:

$$
\left\{\begin{aligned}
Y & >0 \\
P_{1} A_{11}+A_{11}^{T} P_{1}+B_{1}^{0} X B_{1}^{0 T} & +\alpha_{120}^{2} C_{1}^{0} P_{1} Y^{-1} P_{1} C_{1}^{0 T}<0 \\
X & >0 \\
P_{2} A_{22}+A_{22}^{T} P_{2}+B_{2}^{0} Y B_{2}^{0 T} & +\alpha_{210}^{2} C_{2}^{0} P_{2} X^{-1} P_{2} C_{2}^{0 T}<0
\end{aligned}\right.
$$

Since $0<\beta_{1} \leq \alpha_{120}, 0<\beta_{2} \leq \alpha_{210}$, thus $0<\beta_{1}^{2} \leq \alpha_{120}^{2}$, $0<\beta_{2}^{2} \leq \alpha_{210}^{2}$, so we have

$$
\begin{aligned}
& P_{1} A_{11}+A_{11}^{T} P_{1}+B_{1}^{0} X B_{1}^{0 T}+\beta_{1}^{2} C_{1}^{0} P_{1} Y^{-1} P_{1} C_{1}^{0 T}<0 \\
& P_{2} A_{22}+A_{22}^{T} P_{2}+B_{2}^{0} Y B_{2}^{0 T}+\beta_{2}^{2} C_{2}^{0} P_{2} X^{-1} P_{2} C_{2}^{0 T}<0
\end{aligned}
$$

use Schur complement, then (13) holds. This completes the proof.

Remark 4. From the Theorem above, we know that we only need to consider full rank decompositions among the different decompositions of $A_{12}, A_{21}$ under the minimal connection coefficients. From the proof of the Theorem 1 in [32], we know that $\left\|C_{1}\left(s I-A_{11}\right)^{-1} B_{1}\right\|_{\infty} \times$ $\left\|C_{2}\left(s I-A_{22}\right)^{-1} B_{2}\right\|_{\infty}$ can be minimized among different decompositions of $A_{12}, A_{21}$ by LMI method, if we let

$$
\min \left(\left\|C_{1}\left(s I-A_{11}\right)^{-1} B_{1}\right\|_{\infty} \times\left\|C_{2}\left(s I-A_{22}\right)^{-1} B_{2}\right\|_{\infty}\right)=\eta_{0}
$$

we can get $\max \left|\alpha_{12} \alpha_{21}\right|=\eta_{0}^{-1}$.

From the proof above, we can give the following algorithm to get the estimation of $\max \alpha_{12}, \max \alpha_{21}$ for any fixed full rank decompositions.

Step 1. For any fixed full rank decompositions $A_{12}=B_{1}^{0} C_{2}^{0}, A_{21}=B_{2}^{0} C_{1}^{0}$, solve the LMI (13) if it holds, go to step 2; otherwise, stop.

Step 2. Solving the following LMIs:

$$
\begin{aligned}
& {\left[\begin{array}{cc}
P_{1} A_{11}^{T}+A_{11} P_{1}+B_{1}^{0} X B_{1}^{0 T} & P_{1} C_{1}^{0 T} \\
C_{1}^{0} P_{1} & -\frac{1}{\gamma_{2}^{2}} Y
\end{array}\right]<0} \\
& {\left[\begin{array}{cc}
P_{2} A_{22}^{T}+A_{22} P_{2}+B_{2}^{0} Y B_{2}^{0 T} & P_{2} C_{2}^{0 T} \\
C_{2}^{0} P_{2} & -\frac{1}{\gamma_{1}^{2}} X
\end{array}\right]<0}
\end{aligned}
$$


If it holds, then, we can get:

$$
\max \alpha_{12}=\gamma_{1}, \max \alpha_{21}=\gamma_{2}
$$

Otherwise, go to step 3.

Step 3. Choose the appropriate step size $\Delta \beta_{1}, \Delta \beta_{2}$ and move one step size for the LIM (13), i.e., solve the following inequalities:

$$
\begin{aligned}
& {\left[\begin{array}{cc}
P_{1} A_{11}^{T}+A_{11} P_{1}+B_{1}^{0} X B_{1}^{0 T} & P_{1} C_{1}^{0 T} \\
C_{1}^{0} P_{1} & -\frac{1}{\left(\beta_{2}+\Delta \beta_{2}\right)^{2}} Y
\end{array}\right]<0} \\
& {\left[\begin{array}{cc}
P_{2} A_{22}^{T}+A_{22} P_{2}+B_{2}^{0} Y B_{2}^{0 T} & P_{2} C_{2}^{0 T} \\
C_{2}^{0} P_{2} & -\frac{1}{\left(\beta_{1}+\Delta \beta_{1}\right)^{2}} X
\end{array}\right]<0}
\end{aligned}
$$

If it does not hold, stop and get

$$
\max \alpha_{12} \in\left(\beta_{1}, \beta_{1}+\Delta \beta_{1}\right), \max \alpha_{21} \in\left(\beta_{2}, \beta_{2}+\Delta \beta_{2}\right)
$$

Otherwise, keep on moving one step size for the LIM (14) and solve the corresponding inequalities and continue the following process in step 3 . If it moves the $n$ step size, we can get:

$$
\begin{aligned}
& \max \alpha_{12} \in\left(\beta_{1}+(n-1) \Delta \beta_{1}, \beta_{1}+n \Delta \beta_{1}\right) \\
& \max \alpha_{21} \in\left(\beta_{2}+(n-1) \Delta \beta_{2}, \beta_{2}+n \Delta \beta_{2}\right)
\end{aligned}
$$

Obviously, (13) is only a sufficient condition, but it is easy to establish an LMI algorithm for designing decentralized control $F_{1}, F_{2}$.

Theorem 3. For any fixed full rank decompositions $A_{12}=B_{1}^{0} C_{2}^{0}, A_{21}=B_{2}^{0} C_{1}^{0}$, there exist $F_{1}, F_{2}$ and $\alpha_{12}, \alpha_{21}$ as in (10) and decomposition $A_{12}=B_{1} C_{2}, A_{21}=B_{2} C_{1}$ such that (9) holds, if and only if there exist positive $P_{1}, P_{2}, X_{1}, X_{2}$ and any matrices $Y_{1}, Y_{2}$ such that

$$
\begin{aligned}
& {\left[\begin{array}{cc}
P_{1} A_{11}^{T}+A_{11} P_{1}+B_{11} Y_{1}+Y_{1}^{T} B_{11}^{T}+B_{1}^{0} X_{1} B_{1}^{0 T} & P_{1} C_{1}^{0 T} \\
C_{1}^{0} P_{1} & -\frac{1}{\beta_{2}^{2}} X_{2}
\end{array}\right]<0} \\
& {\left[\begin{array}{cc}
P_{2} A_{22}^{T}+A_{22} P_{2}+B_{22} Y_{2}+Y_{2}^{T} B_{22}^{T}+B_{2}^{0} X_{2} B_{2}^{0 T} & P_{2} C_{2}^{0 T} \\
C_{2}^{0} P_{2} & -\frac{1}{\beta_{1}^{2}} X_{1}
\end{array}\right]<0}
\end{aligned}
$$

and decentralized controllers gain are given by $F_{1}=$ $Y_{1} P_{1}^{-1}, F_{2}=Y_{2} P_{2}^{-1}$.

Remark 5. LMIs can be solved easily by using the toolbox [33]. Compare to the result in the Subsection 3.1, result in this section is only sufficient condition, but it is easier to establish an LMI algorithm for designing decentralized control and more simple to compute.

\section{Synergic Stabilization for the Special Dynamical Network}

In this section, we discuss the HSSP for the special model
(6).

\subsection{Nyquist Criterion Method}

For stability analysis of network (6), we show the following to be true.

Theorem 4. There exist connection coefficient gain matrix $K=\left[k_{i j}\right], k_{i j} \in R$ such that $I_{n} \otimes A_{O}+K \otimes A_{C}$ is stable if and only if there exist $\lambda_{i} \in R$ such that $A_{O}+\lambda_{i} A_{C}$ are stable simultaneously for $i=1,2, \ldots, n$.

Proof. Let $P \in R^{n \times n}$ be a nonsingular matrix such that $P^{-1} K P=J$ and $J$ is the Jordan standard form of $K$.Then, based on the Properties of the matrix Kronecker product, we can get:

$$
\begin{aligned}
& \left(P \otimes I_{n_{1}}\right)\left(I_{n} \otimes A_{O}+K \otimes A_{C}\right)\left(P^{-1} \otimes I_{n_{1}}\right) \\
& =I_{n} \otimes A_{O}+J \otimes A_{C}
\end{aligned}
$$

Since the Jordan form matrix $J$ is block upper-triangular, the stability of this system is equivalent to the stability of the $n$ systems defined in the diagonal blocks. For $J \otimes A_{C}$, the diagonal blocks are each $\lambda_{i} A_{C}$, and then we can get the conclusion. This completes the proof.

Remark 6. If $k_{i j}$ need to meet some constraints, then, $\lambda_{i}$ also should satisfy some constraints correspondingly. For example in the consensus or formation control problem:

$$
k_{i j}=\left\{\begin{array}{cc}
-\sum_{q=1, k \neq i}^{n} k_{i q} & j=i \\
k_{i j} \in R & j \neq i
\end{array}\right.
$$

Then, we need that there must exist $\lambda_{i_{0}}=0$ such that $A_{O}+\lambda_{i_{0}} A_{C}$ is stable, i.e., $A_{O}$ is stable, and the associated eigenvector of $\lambda_{i_{0}}$ is $\left(\begin{array}{lll}1 & \ldots & 1\end{array}\right)^{T}$.

In the following discussion, we suppose that $A_{C}=$ $B_{1} C_{1}, C_{1} \in R_{m}^{m \times n}$. We can get the following result.

Theorem 5. There exists Hadamard synergic control matrix pair $\left(K=\left[k_{i j}\right], k_{i j} \in R, F\right)$ such that the network (6) is stable if and only if there exist $\lambda_{i} \in R, i=1,2, \ldots, n$ such that the controller $u_{i}=F y_{i}+z_{i}$ simultaneously stabilizes the set of the following $n$ systems:

$$
\begin{aligned}
\dot{x}_{i} & =A_{O} x_{i}+B_{1} u_{i} \\
y_{i} & =x_{i} \\
z_{i} & =\lambda_{i} C_{1} x_{i}
\end{aligned}
$$

Proof. Using the same transform method as in the Theorem 4, we can get that the network (6) is stable if and only if the following $n$ systems is stable simulta- 
neously if $A_{C}=B_{1} C_{1}$.

$$
\dot{\bar{x}}_{i}=\left(A_{O}+\lambda_{i} B_{1} I C_{1}+B_{1} I F\right) \bar{x}_{i}
$$

Where $\bar{x}=\left(P^{-1} \otimes I_{n_{1}}\right) x$.

This is equivalent to the controller $u_{i}=F y_{i}+z_{i}$ stabilizes the set of the $n$ systems as in (16). This completes the proof.

Remark 7. Theorem 5 reveals that the special network (6) can be analyzed for stability by analyzing the stability of a single system with the same dynamics, modified by only a scalar, representing the interconnection, that take values according to the eigenvalues of the connection coefficient gain matrix $K$.

Hereafter, we refer to the transfer function from $u_{i}$ to $y_{i}$ as $G(s)$; the closed loop system can be shown as Figure 3 in this case. If $G(s)$ is single-input-single-output (SISO), we can state a second version of Theorem 5 which is useful for stability analysis.

Theorem 6. Suppose $G(s)$ is SISO and $p$ is the number of right-half plant poles of $P(s)$. Then, the closed loop system as in Figure 3 is stable if and only if

1) If $\lambda_{i} \neq 0, i=1,2, \ldots, n$, then, the counterclockwise net encirclement of $\left(-\lambda_{i}^{-1}, j 0\right)$ by the Nyquist plot of $-C_{1} P(s)$ is equal to $p$ for $i=1,2, \ldots, n$.

2) Otherwise, $p=0$ and this net encirclement is equal to zero.

Proof. The Nyquist criterion states that the stability of the closed loop system in Figure 3 is equivalent to the number of counterclockwise encirclements of $(-1, j 0)$ by the forward loop $\lambda_{i} C_{1} P(j \omega)$ being equal to the number of the right-half plant poles of $P(s)$, which is assumed to be $p$. This criterion is equivalent to the number of encirclements of $\left(-\lambda_{i}^{-1}, j 0\right)$ by the Nyquist plot of $-C_{1} P(s)$ being $p$. This completes the proof.

Similarly, if $G(s)$ is MIMO, we can give the following result.

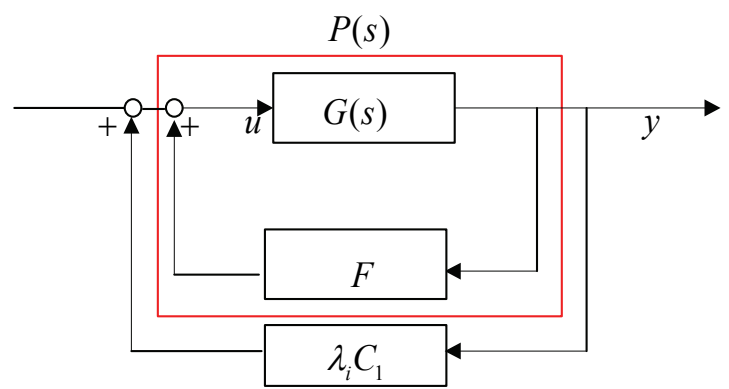

Figure 3. The closed loop of the system (16).
Corollary 1. Suppose $G(s)$ is MIMO and $p$ is the number of right-half plant poles of $P(s)$. Then, the closed loop system as in Figure 3 is stable if and only if

1) If $\lambda_{i} \neq 0, i=1,2, \ldots, n$, then, the counterclockwise net encirclement of the origin by the Nyquist plot of $\operatorname{det}\left(I+\lambda_{i} C_{1} P(s)\right)$ is equal to $p$ for $i=1,2, \ldots, n$.

2) Otherwise, $p=0$ and this net encirclement is equal to zero.

Remark 8. The zero eigenvalue of $K$ can be interpreted as the unobservability of absolute motion in the measurements $z_{i}$. The design strategy in the Theorem 6 can be interpreted as follows: firstly, close the inner loop around $y_{i}$ such that the internal closed loop system $P(s)$ has $p$ right-half plant poles which is equal to the number of uncontrollable poles of the system (16); secondly, close the outer loop around $z_{i}$ such that the whole network system is stable. This can be seen as the synergic action between the feedback control and the connection gain regulation.

\subsection{Algebraic condition}

In this section, we consider the Hadamard synergic stability problem by using the algebraic method. First, we give the following Lemma.

Lemma 1. For any matrix $K$,

$$
\left(I_{n} \otimes A_{O}\right)\left(K \otimes A_{C}\right)=\left(K \otimes A_{C}\right)\left(I_{n} \otimes A_{O}\right)
$$

if and only if $A_{O} A_{C}=A_{C} A_{O}$.

Proof. Based on the fact

$$
\left\{\begin{array}{l}
\left(I_{n} \otimes A_{O}\right)\left(K \otimes A_{C}\right)=K \otimes\left(A_{O} A_{C}\right) \\
\left(K \otimes A_{C}\right)\left(I_{n} \otimes A_{O}\right)=K \otimes\left(A_{C} A_{O}\right)
\end{array}\right.
$$

We can get the conclusion directly. This completes the proof.

Lemma 2. [20] Let $S, T \in C^{n \times n}$ and $S T=T S$, $\lambda_{1}, \ldots, \lambda_{n}, \mu_{1}, \ldots, \mu_{n}$ are their eigenvalues respectively. Then, there exists a permutation $i_{1}, \ldots, i_{n}$ of $1,2, \ldots, n$ such that $\lambda_{1}+\mu_{i_{1}}, \ldots, \lambda_{n}+\mu_{i_{n}}$ are eigenvalues of $S+T$.

Remark 9. Lemma 2 implies that if $S T=T S$, then $\lambda(S+T) \subset \lambda(S)+\lambda(T)$, i.e.

$$
\begin{aligned}
& \max (\operatorname{Re} \lambda(S+T)) \leq \max (\operatorname{Re} \lambda(S))+\max (\operatorname{Re} \lambda(T)) \\
& \min (\operatorname{Re} \lambda(S+T)) \geq \min (\operatorname{Re} \lambda(S))+\min (\operatorname{Re} \lambda(T))
\end{aligned}
$$

Here, $\lambda(X)$ denotes the eigenvalue of matrix $X$.

Theorem 7. Suppose $A_{O} A_{C}=A_{C} A_{O}$ and satisfied

1) If $\max \left(\operatorname{Re} \lambda\left(A_{C}\right)\right) \leq 0$, then, 


$$
\begin{gathered}
\operatorname{Re} \lambda(K)>\max \left\{\frac{-\max \left(\operatorname{Re} \lambda\left(A_{O}\right)\right)}{\max \left(\operatorname{Re} \lambda\left(A_{C}\right)\right)}, 0\right\} \text { or } \\
\frac{-\max \left(\operatorname{Re} \lambda\left(A_{O}\right)\right)}{\min \left(\operatorname{Re} \lambda\left(A_{C}\right)\right)}<\operatorname{Re} \lambda(K)<0
\end{gathered}
$$

2) If $\min \left(\operatorname{Re} \lambda\left(A_{C}\right)\right) \geq 0$, then,

$$
\begin{gathered}
\operatorname{Re} \lambda(K)<\min \left\{\frac{-\max \left(\operatorname{Re} \lambda\left(A_{O}\right)\right)}{\min \left(\operatorname{Re} \lambda\left(A_{C}\right)\right)}, 0\right\} \text { or } \\
0<\operatorname{Re} \lambda(K)<\frac{-\max \left(\operatorname{Re} \lambda\left(A_{O}\right)\right)}{\max \left(\operatorname{Re} \lambda\left(A_{C}\right)\right)}
\end{gathered}
$$

3) If $\max \left(\operatorname{Re} \lambda\left(A_{C}\right)\right)>0$, then,

$$
\begin{aligned}
& \min \left(\operatorname{Re} \lambda\left(A_{C}\right)\right)<0,0<\operatorname{Re} \lambda(K)<\frac{-\max \left(\operatorname{Re} \lambda\left(A_{O}\right)\right)}{\min \left(\operatorname{Re} \lambda\left(A_{C}\right)\right)} \\
& \min \left(\operatorname{Re} \lambda\left(A_{C}\right)\right)>0,0<\operatorname{Re} \lambda(K)<\frac{-\max \left(\operatorname{Re} \lambda\left(A_{O}\right)\right)}{\min \left(\operatorname{Re} \lambda\left(A_{C}\right)\right)}
\end{aligned}
$$

Proof. Based on the Lemma 1 and Lemma 2, we have that

$$
\begin{aligned}
& \max \left(\operatorname{Re} \lambda\left(I_{n} \otimes A_{O}+K \otimes A_{C}\right)\right) \\
& \leq \max \left(\operatorname{Re} \lambda\left(I_{n} \otimes A_{O}\right)\right)+\max \left(\operatorname{Re} \lambda\left(K \otimes A_{C}\right)\right) \\
& =\max \left(\operatorname{Re} \lambda\left(A_{O}\right)\right)+\max \left(\operatorname{Re} \lambda(K) \operatorname{Re} \lambda\left(A_{C}\right)\right)
\end{aligned}
$$

then, we can get the conclusion directly. This completes the proof.

Corollary 2. Suppose that the connection gain matrix $K=\left[k_{i j}\right]$ meet constraint (15), $A_{O} A_{C}=A_{C} A_{O}$ and $A_{O}$ is stable.

1) If $\max \left(\operatorname{Re} \lambda\left(A_{C}\right)\right) \leq 0$, then, $I_{n} \otimes A_{O}+K \otimes A_{C}$ is stable for any $K$ satisfied $k_{i j} \leq 0$.

2) If $\min \left(\operatorname{Re} \lambda\left(A_{C}\right)\right) \geq 0$, then, $I_{n} \otimes A_{O}+K \otimes A_{C}$ is stable for any $K$ satisfied $k_{i j} \geq 0$.

Proof. Based on the Gerschgorin disk theorem, we know that all the eigenvalues of $K=\left[k_{i j}\right]$ are located in the union of the $n$ disk:

$$
G_{i}=\left(z \in R|| z-k_{i i}\left|\leq \sum_{j=1}^{n}\right| k_{i j} \mid\right)
$$

thus, we can get that all the eigenvalues of $K=\left[k_{i j}\right]$ are positive except zero when $k_{i j} \leq 0$ and are negative except zero when $k_{i j} \geq 0$. Then based on the Theorem 7, we can get the conclusion directly. This completes the proof.
When we consider the common decentralized controller $u_{i}=F x_{i}$, if we want to use the conclusions above, we must require that $\left(A_{O}+B_{1} F\right) A_{C}=A_{C}\left(A_{O}+B_{1} F\right)$, this is difficult to solve. Thus, we consider the special case that $A_{C}=a I_{n}, a \neq 0$ and can get the following result.

Corollary 3. If $\left(A_{O}, B_{1}\right)$ is controllable, then for any $K$ there must exist common decentralized controller $u_{i}=F x_{i}$ such that $I_{n} \otimes\left(A_{O}+B_{1} F\right)+K \otimes\left(a I_{n}\right)$ is stable; otherwise, suppose $T A_{O} T^{-1}=\left[\begin{array}{cc}A_{1} & A_{2} \\ 0 & A_{3}\end{array}\right]$, then, there exist common decentralized controller $u_{i}=F x_{i}$ such that $I_{n} \otimes\left(A_{O}+B_{1} F\right)+K \otimes\left(a I_{n}\right)$ is stable if and only if $\lambda(K) \leq \frac{\max \left(\operatorname{Re} \lambda\left(A_{3}\right)\right)}{a}$ for $a>0$ or $\lambda(K) \geq \frac{\max \left(\operatorname{Re} \lambda\left(A_{3}\right)\right)}{a}$ for $a<0$.

Proof. From the fact that for any matrix $K, F$

$$
\begin{aligned}
& \left(I_{n} \otimes\left(A_{O}+B_{1} F\right)\right)\left(K \otimes\left(a I_{n}\right)\right) \\
& =\left(K \otimes\left(a I_{n}\right)\right)\left(I_{n} \otimes\left(A_{O}+B_{1} F\right)\right)
\end{aligned}
$$

and based on the Lemma 2 we can get the conclusion directly.

Remark 10. Based on the analysis in the Corollary 3 for this special case, synergic action between the decentralized feedback control and the connection gain regulations can be interpret as follows, that is: designing the common decentralized controller $u_{i}=F x_{i}$ to stabilize the controllable part firstly, and designing connection coefficient gain matrix $K$ to stabilize the uncontrollable part secondly.

\subsection{Network with Communication Time-Delays}

In this section, we consider a network of continuous-time integrators in which the $i$-th subsystem state $x_{i}$ passes through a communication channel $e_{i j}$ with time-delay $\tau_{i j}>0$ before getting to $j$-th subsystem. The transfer function associated with the edge $e_{i j}$ can be expressed as: $h_{i j}(s)=e^{-\tau_{i j} s}$ in the Laplace domain. As the discussion in [34], to gain further insight in the relation between the connection gain matrix $K$ and the maximum time-delays, we focus on the simplest possible case where the time-delays in all channels are equal to $\tau>0$ and $h_{i j}(s)=h(s)=e^{-\tau s}$. Then the network system can be written as:

$$
\dot{x}_{i}(t)=A_{O} x_{i}(t-\tau)+\sum_{j=1}^{n} k_{i j} A_{C}\left(x_{j}(t-\tau)\right)+B_{1} u_{i}
$$

After taking the Laplace transform of both sides, we 
can get

$$
s X_{i}(s)-x_{i}(0)=h(s) A_{O} X_{i}(s)+\sum_{j=1}^{n} h(s) k_{i j} A_{C}\left(X_{j}(s)\right)
$$

The set of equations above can be rewritten in a compact form as:

$$
X(s)=\left(s I-h(s)\left(I_{n} \otimes A_{O}+K \otimes A_{C}\right)\right)^{-1} x(0)
$$

The convergence analysis for a network of integrator nodes with communication time-delays reduces to stability analysis for a multiple-input-multiple-output (MIMO) transfer function:

$$
G(s)=\left(s I-h(s)\left(I_{N} \otimes A_{O}+K \otimes A_{C}\right)\right)^{-1}
$$

In the following, we give the stability result of the model (17).

Theorem 8. Consider a network of integrator nodes with equal communication time-delay $\tau>0$ in all links. Assume the matrix $I_{n} \otimes A_{O}+K \otimes A_{C}$ has no eigenvalue of zero or the algebraic multiplicity of the zero eigenvalue is 1 . Then the model (17) is stable if and only if either of the following equivalent conditions are satisfied:

1) $\tau \in\left(0, \tau_{0}\right)$ with $\tau_{0}=\min \left(\alpha_{i}\right)$, where

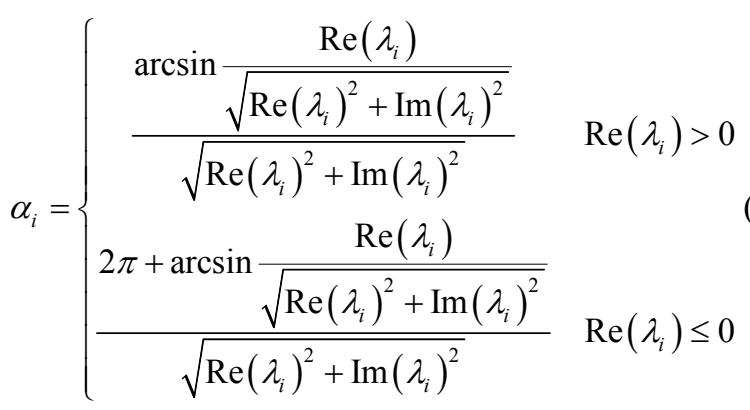

$\lambda_{i}$ is the eigenvalue of the matrix $I_{n} \otimes A_{O}+K \otimes A_{C}$.

2) The Nyquist plot of $\Gamma(s)=\frac{e^{-\tau s}}{s}$ has a zero encirclement around $\frac{1}{\lambda_{i}}$ for $\lambda_{i} \neq 0$.

Proof. To establish the stability of (17), we use frequency domain analysis. We have $X(s)=G(s) x(0)$. Define $H(s)=G^{-1}(s)=s I+h(s)\left(I_{n} \otimes A_{O}+K \otimes A_{C}\right)$. Then, we require that all the zeros of $\operatorname{det}(H(s))$ are on the Left Hand Plane (LHP) or $s=0$. Let $\omega_{i}$ be the normalized eigenvector of $I_{n} \otimes A_{O}+K \otimes A_{C}$ associated with the eigenvalue $\lambda_{i}$. If the matrix $I_{n} \otimes A_{O}+K \otimes A_{C}$ has zero eigenvalue and suppose $\lambda_{1}=0$, then $s=0$ in the direction $\omega_{1}$ is a zero of $\operatorname{det}(H(s))$ since
$H(0) \omega_{1}=\left(I_{n} \otimes A_{O}+K \otimes A_{C}\right) \omega_{1}=0 ;$ otherwise $s=0$ is not a zero of $\operatorname{det}(H(s))$.

Furthermore, we can get that any eigenvector of $H(s)$ is an eigenvector of $I_{n} \otimes A_{O}+K \otimes A_{C}$ and vice verse. Then, we can get that for any $s$ of the zero of $\operatorname{det}(H(s))$, we must have $H(s) \omega_{i}=0$ for some one $i$, i.e.,

$$
\begin{aligned}
& H(s) \omega_{i}=\left(s I+h(s)\left(I_{n} \otimes A_{O}+K \otimes A_{C}\right)\right) \omega_{i} \\
& =\left(s+\lambda_{i} e^{-\tau s}\right) \omega_{i}=0
\end{aligned}
$$

But $\omega_{i} \neq 0$, thus, $s \neq 0$ satisfies the following equation:

$$
\frac{1}{\lambda_{i}}+\frac{e^{-\tau s}}{s}=0
$$

Thus, if the net encirclement of the Nyquist plot of $\Gamma(s)=\frac{e^{-\tau s}}{s}$ around $-\frac{1}{\lambda_{i}}$ for $\lambda_{i} \neq 0$ is zero, then all the poles of $G(s)$ except $s=0$ are stable.

We calculate the upper bound on time-delay $\tau$ as follows. We want to find the smallest value of the time-delay $\tau>0$ such that $\operatorname{det}(H(s))$ has a zero on the imaginary axis. Set $s= \pm j \omega$ in (19), we can get

$$
\begin{aligned}
& j \omega+e^{-j \omega \tau} \lambda_{i}=0 \\
& -j \omega+e^{j \omega \tau} \lambda_{i}=0
\end{aligned}
$$

multiplying both sides of the two equations above, we get $\omega^{2}+\lambda_{i}^{2}-2 \omega \lambda_{i} \sin (\omega \tau)=0$.

Let $\lambda_{i}=\operatorname{Re}\left(\lambda_{i}\right)+j \operatorname{Im}\left(\lambda_{i}\right)$, then, we have

$$
\begin{aligned}
& \left(\omega-\operatorname{Re}\left(\lambda_{i}\right)\right)+2 \omega \operatorname{Re}\left(\lambda_{i}\right)(1-\sin (\omega \tau))-\operatorname{Im}\left(\lambda_{i}\right) \\
& +2 j \omega\left(\operatorname{Re}\left(\lambda_{i}\right) \operatorname{Im}\left(\lambda_{i}\right)-\operatorname{Im}\left(\lambda_{i}\right) \sin (\omega \tau)\right)=0
\end{aligned}
$$

Assume $\omega>0$ (due to $s \neq 0$ ), then from the equation above, we can get:

$$
\omega=\sqrt{\left(\operatorname{Re} \lambda_{i}\right)^{2}+\left(\operatorname{Im} \lambda_{i}\right)^{2}}, \operatorname{Re}\left(\lambda_{i}\right)=\omega \sin (\omega \tau)
$$

This implies

$$
\tau\left(\sqrt{\left(\operatorname{Re} \lambda_{i}\right)^{2}+\left(\operatorname{Im} \lambda_{i}\right)^{2}}\right)=2 k \pi+\arcsin \frac{\operatorname{Re}\left(\lambda_{i}\right)}{\sqrt{\operatorname{Re}\left(\lambda_{i}\right)^{2}+\operatorname{Im}\left(\lambda_{i}\right)^{2}}},
$$

i.e.

$$
\tau=\frac{2 k \pi+\arcsin \frac{\operatorname{Re}\left(\lambda_{i}\right)}{\sqrt{\operatorname{Re}\left(\lambda_{i}\right)^{2}+\operatorname{Im}\left(\lambda_{i}\right)^{2}}}}{\sqrt{\operatorname{Re}\left(\lambda_{i}\right)^{2}+\operatorname{Im}\left(\lambda_{i}\right)^{2}}}
$$


thus, the smallest $\tau>0$ satisfied that $\tau_{0}=\min \left(\alpha_{i}\right)$ and $\alpha_{i}$ are given in (18).

Due to the continuous dependence of the roots of (19) in $\tau$ and the fact that all the zeros of this equation except $s=0$ for $\tau=0$ are located on the open LHP, for all $\tau \in\left(0, \tau_{0}\right)$, the roots of (19) are on the open LHP, and therefore the poles of $G(s)$ are all stable except $s=0$, but the algebraic multiplicity of the zero eigenvalue is 1 . We can repeat a similar argument for the assumption that $\omega<0$. This completes the proof.

Remark 11. From the condition (1) of the Theorem above, we can see that the upper bound on time-delay $\tau_{0}$ is determined by the eigenvalues $I_{n} \otimes A_{O}+K \otimes A_{C}$. Thus, if $A_{O} A_{C}=A_{C} A_{O}$, then based on the Lemma 2, we can design the desired connection gain matrix $K$ in order to obtain expected upper bound on time-delay.

\section{Conclusions}

In this paper, Hadamard synergic stabilization problem is investigated. Synergic stabilization problem for a special kind of networks are studied by using the Nyquist criterion. The mechanism of the synergic action between two control strategies: feedback control and the connection coefficients regulations are presented. Networks with communication time-delays are also discussed. Furthermore, synergic stabilization problem for the general dynamical network composed of two subsystems are investigated. The regulations of the interconnections can be exploited to improve the stability of the closed-loop system. It should be noted that only some special network models have been investigated in this paper, many more general network models remain to be challenging subjects for future research. Although Hadamard synergic control problem has not received much attention, we suggest that it will probably turn out to be widespread in power electrical engineering and the epidemic control system. We hope that our work will stimulate further studies of this new kind of control problem.

\section{References}

[1] X. F. Wang, "Complex Networks: Topology, Dynamics, and Synchronization," International Journal of Bifurcation and Chaos, Vol. 5, No. 12, 2002, pp. 885-916.

[2] D. J. Watts and S. H. Strogatz, "Collective Dynamics of 'Small-World' Networks," Nature, Vol. 393, No. 6684, 1998, pp. 440-442.

[3] X. Li, X. F. Wang and G. R. Chen., "Pinning a Complex Dynamical Network to its Equilibrium," IEEE Transactions on Circuits and Systems-I, Vol. 51, No. 10, 2004, pp. 2074-2085.
[4] V. Lesser, C. L. Ortiz and M. Tamb, "Distributed Sensor Networks: A Multiagent Perspective," Kluwer Academic Publishers, Boston, 2003.

[5] S. S. Mascolo, "Congestion Control in High-Speed Communication Networks Using the Smith Principle," Automatica, Vol. 35, No. 11, 1999, pp. 1921-1935.

[6] L. Huang and Z. S. Duan, "Complexity in Control Science," Acta Automatica Sinica, Vol. 29, No. 5, 2003, pp. 748-753.

[7] Z. S. Duan, L. Huang, J. Z. Wang and L. Wang, "Harmonic Control between Two Systems," Acta Automatica Sinica, Vol. 129, No. 1, 2003, pp. 14-21.

[8] Z. S. Duan, J. Z. Wang and L.Huang, "Special Decentralized Control Problems and Effectiveness of Parameter-Depend Lyaounov Function Method," Proceedings of the American Control Conference, Boston, 2005, pp. 1697-1702.

[9] Y. Zou, M. H. Yin and H. D. Chiang, "Theoretical Foundation of Controlling U.E.P Method in Network Structure Preserving Power System Model," IEEE Transactions on Circuits and Systems I, Vol. 50, No. 10, 2003, pp. 13241336.

[10] K. Sun, D. Z. Zheng and Q. Lu, "A Simulation Study of OBDD-Based Proper Splitting Strategies for Power Systems, under Consideration of Transient Stability," IEEE Transaction on Power Systems, Vol. 120, No. 1, 2005, pp. 389-399.

[11] K. Sun, D. Z. Zheng and Q. Lu, "Splitting Strategies for Islanding Operation of Large-Scale Power Systems Using OBDD-Based Methods," IEEE Transactions on Power Systems, Vol. 18, No. 2, 2003, pp. 912-923.

[12] Q. C. Zhao, K. Sun, D. Z. Zheng, J. Ma and Q. Lu, "A Study of System Splitting Strategies for Island Operation of Power System: A Two-Phase Method Based on OBDDs," IEEE Transactions on Power Systems, Vol. 18, No. 4, 2003, pp. 1556-1565.

[13] X. F. Yan, Y. Zou and J. Li, "Optimal Quarantine and Isolation Strategies in Epidemics Control," World Journal of Modeling and Simulation, Vol. 3, No. 3, 2007, pp. 202-211.

[14] X. F. Yan and Y. Zou, "Optimal Control for Internet Worm,” ETRI Journal, Vol. 30, No. 1, 2008, pp. 81-88.

[15] X. F. Yan and Y. Zou, "Isolation Treatment Strategy for Emergency Control," Journal of systems Engineering, Vol. 24, No. 2, 2009, pp. 129-135.

[16] Y. Zou, "Investigation on Mechanism and Extension of Feedback: An Extended Closed-loop Coordinate Control of Interconnection-regulation and Feedback," Journal of Nanjing University of Science and Technology, Vol. 34, No. 1, 2010, pp. 1-7.

[17] K. J. Astrom, "Limitations on Control System Performance," European Journal of Control, Vol. 4, No. 1, 2000, pp. 2-20.

[18] R. M. Murray, K. J. Astrom, S. P. Boyd, et al., "Future Directions in Control in an Information-Rich World," IEEE Control Systems Magazine, Vol. 23, No. 2, 2003, pp. 20-33. 
[19] L. Guo, "Exploring the Maximum Capability of Adaptive Feedback," International Journal of Adaptive Control and Signal Processing, Vol. 16, No. 1, 2002, pp. 341354.

[20] J. L. Chen and X. H. Chen, "Special Matrices," Tsinghua Publisher, Beijing, 2001.

[21] X. J. Liu and Y. Zou, "On the Hadamard Synergic Stabilization Problem: The Stabilization Via a Composite Strategy of Connection-Regulation and Feedback Control: Matrix Inequality Approach," International Journal of Innovative Computing, Information and Control, Vol. 6, No. 5, 2010, pp. 2383-2392.

[22] Z. S. Duan, J. Z. Wang, G. R. Chen and L. Huang, "Stability Analysis and Decentralized Control of a Class of Complex Dynamical Networks," Automatica, Vol. 44, No. 4, 2008, pp. 1028-1035.

[23] A. B. Gumel, S. Ruan, T. Day, et al., "Modeling Strategies for Controlling SARS Outbreaks," Proceedings of the Royal Society, Vol. 49, No. 9, 2004, pp. 1465-1476.

[24] X. Zhang, X. Hu and Z. Bao, "Blind Source Separation Based on Grading Learning," Science in China-Series E, Vol. 32, No. 5, 2002, pp. 693-793.

[25] S. Chen, "A Lower Bound for the Minimum Eigenvalue of the Hadamard Product of Matrices," Linear Algebra and its Applications, Vol. 37, No. 8, 2004, pp. 159-166.

[26] H. Li, T. Huang, S. Shen, et al., "Lower Bounds for the Minimum Eigenvalue of Hadamard Product of an Mmatrix and its Inverse," Linear Algebra and its Applications, Vol. 4, No. 20, 2007, pp. 235-247.
[27] G. Visick, "A Quantitative Version of the Observation that the Hadamard Product is a Principal Submatrix of the Kronecker Product," Linear Algebra and its Applications, Vol. 30, No. 4, 2000, pp. 45-68.

[28] M. Neumann, "A Conjecture Concerning the Hadamard Product of Inverses of $M$-matrices," Linear Algebra and its Applications, Vol. 28, No. 5, 1998, pp. 277-290.

[29] L. Qiu and X. Zhan, "On the Span of Hadamard Products of Vectors," Linear Algebra and its Applications, Vol. 422, No. 1, 2006, pp. 304-307.

[30] J. A. Fax and R. M. Murray, "Information Flow and Cooperative Control of Vehicle Formations," IEEE Transactions on Automatic Control, Vol. 49, No. 9, 2004, pp. 1465-1476.

[31] R. O. Saber and M. Murray, "Consensus Protocols for Networks of Dynamic Agents," Proceedings of the American Control Conference, Denver, 2003, pp. 951-956.

[32] Z. S. Duan, L. Huang, L. Wang and J. Z. Wang, "Some Applications of Small Gain Theorem to Interconnected Systems," Systems and Control Letters, Vol. 52, No. 3-4, 2004, pp. 263-273.

[33] P. Gahinet, A. Nemirovski and A. J. Laub, "LMI Control Toolbox," TheMath Works, Inc., Natick, 1995.

[34] R. O. Saber and M. Murray, "Consensus Problems in Networks of Agents with Switching Topology and TimeDelays," IEEE Transactions on Automatic Control, Vol. 49, No. 9, 2004, pp. 1520-1533. 